\title{
Die schweizerische Entwicklungszusammenarbeit und die Umweltproblematik
}

Rahmenkredit für Umweltprogramme von globaler Bedeutung in Entwicklungsländern

Theo Wiederkehr

\section{(2) OpenEdition}

\section{Journals}

Electronic version

URL: http://journals.openedition.org/sjep/1377

DOI: 10.4000/sjep.1377

ISSN: 1663-9677

Publisher

Institut de hautes études internationales et du développement

\section{Printed version}

Date of publication: 1 janvier 1992

Number of pages: 177-182

ISSN: 1660-5926

\section{Electronic reference}

Theo Wiederkehr, «Die schweizerische Entwicklungszusammenarbeit und die Umweltproblematik », Schweizerisches Jahrbuch für Entwicklungspolitik [Online], 11 | 1992, Online erschienen am: 19 Mai 2013, abgerufen am 08 September 2020. URL : http://journals.openedition.org/sjep/1377 ; DOI : https:// doi.org/10.4000/sjep.1377 


\title{
Die schweizerische Entwicklungszusammen- arbeit und die Umweltproblematik
}

\section{Rahmenkredit für Umweltprogramme von globaler Bedeutung in Entwicklungsländern}

\author{
Theo Wiederkehr
}

\section{Die Problematik}

Der zum Teil massive Wandel und die zahlreichen Konzeptänderungen, denen die Entwicklungszusammenarbeit in den letzten Jahrzehnten unterworfen war, zeugen von der Komplexität der Entwicklungsproblematik. Die Richtungsănderungen sind auch Ausdruck eines zunehmenden Bewusstseins dieser Komplexität und gleichzeitig das Eingeständnis früher gemachter Fehler. Zweifellos haben die Entwicklungsinstitutionen, die Geldgeber und die Entwicklungsländer selbst in der Vergangenheit viel gelernt und vieles wird noch zu lernen sein. Durch die Entspannung zwischen Ost und West sind die Voraussetzungen dazu besser denn je, indem sich das Interesse aller Beteiligten vermehrt auf die eigentlichen Ziele der Entwicklung und die zentralen Bedürfnisse der Entwicklungsländer wird konzentrieren können.

Die Erfahrung zeigte, dass ausschliessliches Projektdenken und eindimensionales Handeln allein bei Weitem nicht genügt, um ein anvisiertes Entwicklungsproblem zu lösen. So entstanden aus Projekten weitergreifende Programme, die 
auch die Probleme oben- und unterhalb anzupacken und damit den sektoriellen Gesamtrahmen zu verbessern suchten. Der Programmansatz genügte aber auch dann nicht mehr, wenn zudem der wirtschaftliche, politische, soziale und institutionelle Rahmen nicht stimmte. Der enge innere Zusammenhang zwischen Armut, Bevölkerungszunahme, Verschuldung und Umweltzerstörung, um nur die wesentlichsten Faktoren zu nennen, trat immer klarer zum Vorschein. Einseitige Zielsetzungen zum Beispiel wirtschaftlicher Art führten zu Misserfolgen, wenn diese inneren Zusammenhänge nicht genügend berücksichtigt wurden. Die alarmierende Zerstörung der Umwelt in den Entwicklungsländern ist ein deutliches Zeichen dafür, dass allzu oft andere Entwicklungsziele zu Lasten der Umwelt und mit einem Raubbau an den natürlichen Ressourcen des Landes, angestrebt wurden.

Die Nachhaltigkeit der Entwicklung ist heute ein in der entwicklungspolitischen Debatte anerkannter Grundsatz. Im Bezug auf die Umwelt besagt er, dass langfristige Entwicklung nur unter gleichzeitigem Erhalt der natürlichen Ressourcen erreicht werden kann. Die Oekonomie der natürlichen Ressourcen soll als integraler Bestandteil in die Entwicklungspolitik miteinbezogen werden.

Die weitergehende Abholzung der Tropenwälder, die grossflächige Verarmung und Erosion der Böden, die rasch zunehmende Luftverschmutzung in den Städten und die Vergiftung von Flüssen und Seen lassen deutlich werden, dass Theorie und Praxis in der Realität noch weit auseinanderklaffen. Die Erkenntnis, dass die Entwicklungsländer durch die Zerstörung der Umwett ihrer eigenen Existenzgrundlagen mehr und mehr beraubt werden und damit ihre sozialen und wirtschaftlichen Entwicklungsmöglichkeiten auf eine immer schmäler werdende Basis gestellt werden, hat das Thema "Umwelt und Entwicklung" ins Zentrum des internationalen entwicklungspolitischen Interesses gerückt.

In Anbetracht ihrer schwierigen wirtschaftlichen und finanziellen Situation haben die Entwicklungsländer oft keine andere Wahl als ihr Ueberleben kurzfristig durch weitere Uebernutzung ihrer natürlichen Ressourcen zu sichern. Sie müssen zudem befürchten, von den in erster Linie durch die Industrieländer verursachten globalen Umweltproblemen besonders in Mitleidenschaft gezogen zu werden. Die Belastung der globalen Umweltressourcen hat zweifellos eine obere Grenze erreicht und diese zum Teil bereits überschritten. Das unbestreitbare Anrecht der Entwicklungsländer auf wirtschaftliches Wachstum erfordert deshalb nicht nur eine nachhaltige und sparsamere Nutzung der Ressourcen im Süden, sondern ebensosehr eine Reduktion der Energie- und Rohstoffverschwendung im Norden.

\section{Die Umweltpolitik der schweizerischen Entwicklungszusammenarbeit}

In der an das Parlament gerichteten Botschaft über die Weiterfühnung der technischen Zusammenarbeit und der Finanzhilfe zugunsten von Entwick-lungsländern nimmt die Umweltproblematik eine wichtige Rolle ein. Nach-drücklich wird dabei auf den engen Zusammenhang zwischen Umwelt und Entwicklung hin- 
gewiesen und die Notwendigkeit des Einbezugs der Umweltaspekte in die Entwicklungszusammenarbeit unterstrichen.

Das Gebot der Nachhaltigkeit der Entwicklung erfordert den Einbezug einer Umweltpolitik, welche eine nachhaltige Ressourcennutzung anstrebt und diese mit den wirtschaftlichen und sozialen Entwicklungszielen in Übereinstimmung bringt. Es geht also im Wesentlichen darum, die Umweltvertrăglichkeit neben den verschiedenen anderen Elementen als Kriterium in die Planungs-, Entscheidungs- und Durchführungsprozesse von Entwicklungsvorhaben miteinzubeziehen. Die Integration der Umweltziele in die Entwicklungstätigkeit steht also im Vordergrund und, von Ausnahmen abgesehen, nicht spezifische Umweltverträglichkeitsprüfungen.

Der Fachdienst Umwelt/Forstwirtschaft der Direktion für Entwicklungszusammenarbeit und humanitäre Hilfe DEH ist seit einiger Zeit an der Erarbeitung eines geeigneten Instrumentariums, welches den Verantwortlichen für die Entwicklungsprogramme den Einbezug der Umweltverträglichkeit in ihre Projektarbeit erleichtern soll. Die DEH hat sich zu diesem Zwe-cke der Unterstützung des geographischen Institutes der Universität Bern versichert und beabsichtigt, ab Beginn 1992 die Einführung dieses Instrumentariums mit einem Ausbildungsprogramm zum Thema Umwelt und Entwicklung zu verbinden. Parallel dazu soll eine breite Evaluation der Erfahrung der DEH im Umgang mit Umwelt- bzw. Ressourcenfragen zusätzliche Erkenntnisse und Anhaltspunkte liefern.

Neben diesem Anliegen der Integration der Umwelt in die Entwicklungszusammenarbeit ist eine beträchtliche Anzahl Projekte der DEH direkt auf die Verbesserung der ökologischen Grundlagen ausgerichtet oder weist eine starke ökologische Komponente auf. Letzteres gilt vor allem für eine Reihe von Forst- und Landwirtschaftsprojekten. In Zukunft sollen vermehrt auch Programme zur Stärkung von Institutionen und der Ausbildung sowie der nationalen und internationalen Umweltforschung unterstützt werden. Es ist der DEH ferner ein Anliegen, die Prinzipien der schweizerischen Entwicklungszusammenarbeit in den internationalen Dialog im Umweltbereich, der in Anbetracht des globalen Charakters der Problematik mit zunehmender Intensität geführt wird, einzubringen und den Entwicklungsländern durch die Finanzienung der Teilnahmekosten die gebührende Vertretung ihrer Interessen in den internationalen Gremien zu ermöglichen.

\section{Der Rahnmenkredit von $\mathbf{3 0 0}$ Millionen Franken für Umweltprogramme von globaler Bedeutung in Entwicklunslăndern}

\section{Die Ausgangslage}

Ausgehend von der von schweizerischen Hilfswerken lancierten Petition "Entwicklung braucht Entschuldung" und auf der Grundlage einer Botschaft des Bundesrates hat das Parlament anlässlich der Märzsession 1991 beschlossen, im Rahmen der 700-Jahrfeier der Eidgenossenschaft eine spezielle Geste von 700 Millionen Franken zugunsten der Entwicklungsländer zu machen. Der Betrag 
wurde in zwei Rahmenkredite aufgeteilt, wovon der eine in der Höhe von 400 Millionen Franken für Entschuldungsmassnahmen und der zweite von 300 Millionen Franken zur Finanzienung von Umweltprogrammen und -projekten von globaler Bedeutung in Entwicklungslăndern bestimmt ist.

Die Schweiz hat im Laufe der vergangenen Jahre ihre nach aussen gerichtete Umweltpolitik in verschiedener Hinsicht verstärkt. So war sie beispielsweise Initiantin der Konvention über den grenzüberschreitendem Transport gefährlicher Abfälle, die im März 1989 in Basel verabschiedet wurde. Die Schweiz spielt auch im Zusammenhang mit der Welt-Klimakonferenz und den Verhandlungen über eine internationale Klimakonvention eine sehr aktive Rolle. Das Engagement im internationalen Umweltbereich wird der Schweiz insofern erleichtert, als sie im eigenen Land eine im Quervergleich sehr fortschrittliche Umweltpolitik verfolgt. Durch den obenerwähnten Rahmenkredit von 300 Millionen Franken wird die nach aussen gerichtete Umweltpolitik der Schweiz durch ein Instrument ergänzt, welches den Einbezug der Entwicklungsländer in die weltweiten Anstrengungen zur Bewältigung der globalen Umweltprobleme ermöglichen soll.

\section{Zlele und Inhalt des Rahmenkredites}

Der für eine 5-jährige Verpflichtungsdauer vorgesehene Rahmenkredit soll den Entwicklungsländern ermöglichen, ihrerseits einen Beitrag zur Lösung der globalen Umweltprobleme zu leisten. Obwohl die Entwicklungsländer bisher einen vergleichsweise geringen Anteil an der Verursachung der globalen Umweltprobleme hatten, kommt innen dennoch in jeder Strategie zur Bewältigung dieser Probleme eine ganz wesentliche Rolle zu. Einerseits werden ihre Emissionen infolge des grossen Nachholbedarfs an wirtschaftlicher Entwicklung wachsen, und andererseits nehmen die Entwicklungsländer in verschiedenen Bereichen der globalen Umweltproblematik eine Schlüsselstellung ein. Diese ist am offensichtlichsten im Zusammenhang mit der Zerstörung der Tropenwälder, welche fast ausschliesslich in Entwicklungsländern liegen und im Hinblick auf Klimaveränderung und Erhalt der Biodiversität von ausschlaggebender Bedeutung für die ganze Menschheit sind. Die Entwicklungsländer sind deshalb sowohl als Mitverursacher als auch für die Lösung von globalen Umweltproblemen unerlässliche Partner.

Von den Entwicklungsländern wird also ein Beitrag zur Lösung von Problemen enwartet, die in erster Linie von den reichen Industrieländern verursacht wurden. Die Nutzniesser dieses Beitrages werden alle Menschen sein und nicht prioritär die Entwicklungsländer selbst. In Anbetracht der grossen Entwicklungsbedürfnisse und der sehr knappen Ressourcen der Entwicklungsländer sind deshalb die Industrieländer heute weitgehend bereit, den Entwicklungsländern für deren Beitrag an die Lösung globaler Umweltprobleme zusätzliche Mittel zu den Entwicklungsgeldern zur Verfügung zu stellen. Der Rahmenkredit von 300 Millionen Franken erlaubt der Schweiz, ihren Teil zu diesen Anstrengungen beizutragen. 


\section{Die Verwendung der Mittel}

Im Rahmen von Verhandlungen zu internationalen Abkommen im globalen Umweltbereich sind oft spezifische Finanzierungsmechanismen zum Einbezug der Entwicklungslănder notwendig. Im Zusammenhang mit dem Montreal Protokoll über ozonabbauende Stoffe wurde in diesem Sinne ein erster Fonds geschaffen, durch welchen entsprechende Massnahmen in Entwicklungsländern finanziert werden sollen. Ein wichtiger Schritt in diese Richtung stelt die im Frühjahr 91 entstandene "Global Environment Facility" dar. Der damit verbundene Fonds in der Höhe von rund 2 Milliarden Franken für eine erste Pilotphase von 3 Jahren wird von der Weltbank, UNDP und UNEP gemeinsam verwaltet und zur Finanzierung von Umweltprogrammen von globaler Bedeutung in Ländern mit einem BSP pro Kopf von weniger als 4000 US Dollar (1989) verwendet werden. Die Schweiz leistet daran einen Beitrag von ca. 80 Millionen Franken. Für solche Leistungen und weitere Verpflichtungen der Schweiz im Zusammenhang mit internationalen Fonds im globalen Umweltbereich sind im Rahmenkredit indikativ 120 Millionen Franken vorgesehen. Zuständig für die Verwendung dieser Mittel ist das Bundesamt für Umwelt, Wald und Landschaftschutz gemeinsam mit der Direktion für Internationale Organisationen des EDA.

Die Zuständigkeit für die Durchführung der bilateralen und multi- bilateralen Aktionen, für welche 180 Millionen Franken bestimmt sind, hat der Bundesrat der DEH übertragen. In der Tat werden die Probleme und Aufgaben, die sich bei der Planung und Realisierung der Programme ergeben, ähnlich denjenigen bei der Entwicklungszusammenarbeit sein, obwohl deren Zielsetzung und die jeweilige Ausgangslage voneinander verschieden sind. Es geht aber auch hier vonwiegend um die Uebertragung von fachlichem Wissen und Können, um den Aufbau tragfähiger Institutionen, um Forschung und Ausbildung, um die Entwicklung von Sektorpolitiken, teilweise auch um die Finanzierung von Investitionen usw., also um Aufgaben, für deren Durchführung die DEH bereits über geeignete Instrumente verfügt.

Die Botschaft definiert folgende Problembereiche, in welchen die Finanzierung von Massnahmen unter dem Rahmenkredit möglich ist:

- die Erwärmung der Erdoberfläche durch die Treibhausgase

- der Abbau der stratosphärischen Ozonschicht

- die Zerstörung der Tropenwälder

- der Verlust der Biodiversität

- die Verschmutzung der Meere und der internationalen

Binnengewăsser

- der Transport, die Lagerung und der unkontrollierte Gebrauch gefährlicher Chemikalien und Abfälle

- die grenzüberschreitende Luftverschmutzung.

Das zentrale Element bei der Auswahl von Projekten unter dem Rahmenkredit ist deren Relevanz für die globale Umweltproblematik. Die Aktion muss entweder selbst einen signifikanten Beitrag erbringen oder hat das Potential, als Katalisator für weiterreichende Massnahmen zu wirken. Von wesentlicher Bedeutung ist 
ebenfalls deren Einbettung in einen geeigneten umwelt- und entwicklungspolitischen Rahmen. Obwohl längerfristig eine gewisse geographische Konzentration angestrebt wird, sind prinzipiell Aktionen in allen Entwicklungs- und Schwellenländern möglich.

Die erste Phase der Projektdurchführung wird durch einen gewissen Pragmatismus gekennzeichnet sein, da es sich weitgehend um die Zusammenarbeit mit neuen Partnern und Ländern und um neue Aktionsbereiche handelt. Aufgrund der Erfahrungen wird es anschliessend möglich und notwendig sein, den durch die Botschaft des Bundesrates gegebenen weiten Rahmen präziser zu definieren und die Aktionen auf eine kohärente konzeptionelle und programmatische Grundlage zu stellen. 